\title{
Educação em Saúde para Prevenção do Câncer de Colo do Útero em Mulheres do Município de Santo Ângelo/RS.
}

\author{
Education in Health for Prevention of Uterine Cervical Cancer \\ in Women in Santo Ângelo, State of Rio Grande do Sul, Brazil.
}

Micheli Renata Casarin ${ }^{1}$

Jaqueline da Costa Escobar Piccoli ${ }^{2}$

${ }^{1}$ Curso de Ciências Biológicas, Universidade Regional Integrada do Alto Uruguai e das Missões. BR $472 \mathrm{Km} 592$, CP 118.

97500-970 Uruguaiana RS. michelicasarin@yahoo.com.br ${ }^{2}$ Universidade Federal do

Pampa.

\begin{abstract}
In Brazil, cervical uterine cancer is a major public health problem as it has high mortality rate indices among women of different ages. A cytopathological examination is recommended for preventing this type of cancer from the early stages of a woman's reproductive life. The scope of this study was both to give talks on sexual health education and establish the sexual health profile of women from St. Angelo/RS. The lectures included measures for prevention as well as identification of possible symptoms of the disease. The research into the sexual health profile of the participants was conducted by a structured questionnaire applied to 140 women aged between 15 and 60. It was found that even experiencing difficulties and apprehension, the majority of the women performed the preventive examination. The motivation for taking the exam is the appearance of symptoms and the habit of caring for their health. The participants mentioned the importance of health professionals and educators working together. The study sought to promote health and cancer prevention, seeking to avoid the disease and ensure better living conditions for women.
\end{abstract}

Key words Uterine cervical cancer, Health education, Prevention
Resumo No Brasil, o câncer cérvico-uterino constitui importante problema de saúde, pois apresenta alto indice de letalidade entre mulheres de varias idades. O exame citopatológico foi preconizado como medida de prevenção deste tipo de câncer, devendo ser realizado a partir do início da vida sexual. O estudo teve um caráter prático de promover educação em saúde sexual e conhecer o perfil da saúde sexual de mulheres de Santo Ângelo/RS. As palestras abordaram formas de prevenção e identificação de possíveis sintomas da doença. O levantamento do perfil de saúde sexual das participantes foi realizado através da aplicação de um questionário estruturado. Participaram das palestras 140 mulheres com idades entre 15 e 60 anos. Verificou-se que, mesmo enfrentando dificuldades e medo, a maioria delas realiza exame preventivo, motivada por aparecimento de sintomas e pelo hábito de cuidar da saúde. As participantes referiram a importância da integração entre profissionais e educadores em Saúde. O estudo foi direcionado no sentido de dar relevância à promoção da saúde e à prevenção do câncer, buscando evitar a doença e obter melhores condições de vida para as mulheres.

Palavras-chave Câncer cérvico-uterino, Educação em saúde, Prevenção 


\section{Introdução}

No Brasil, estima-se que o câncer de colo uterino seja a terceira neoplasia maligna mais comum entre as mulheres, sendo apenas superado pelo câncer de pele (não melanoma), e pelo câncer de mama ${ }^{1}$.

A evolução do câncer do colo do útero, na maioria dos casos, se dá de forma lenta, passando por fases pré-clínicas detectáveis e curáveis. Dentre todos os tipos de câncer, é o que apresenta um dos mais altos potenciais de prevenção e cura. Seu pico de incidência situa-se entre mulheres de 40 aos 49 anos de idade, e apenas numa pequena porcentagem, naquelas com menos de 30 anos, sendo que a faixa de idade para detecção precoce é dos 20 aos 29 anos, período que corresponde ao pico de incidências das lesões precursoras da doença e antecede ao pico de mortalidade pelo câncer.

Este tipo de câncer ainda é um problema de saúde pública em países em desenvolvimento, como o Brasil, pois alcança altas taxas de prevalência e mortalidade em mulheres de extratos sociais e econômicos mais baixos. Estudos revelam que existe a associação entre o câncer de colo uterino e o baixo nível socioeconômico em todas as regiões do mundo. Os grupos mais vulneráveis estão onde existem barreiras de acesso à rede de serviços de saúde, para detecção e tratamento da patologia e de suas lesões precursoras, advindas das dificuldades econômicas e geográficas, insuficiência de serviços e por questões culturais, como medo, desconsideração de sintomas importantes e preconceito ${ }^{2}$.

São considerados fatores de risco para o câncer do colo do útero a multiplicidade de parceiros e a história de infecções sexualmente transmissíveis, a idade precoce na primeira relação sexual e a multiparidade. Além desses fatores, estudos epidemiológicos sugerem outros, cujo papel ainda não é conclusivo, tais como tabagismo, alimentação pobre em alguns micronutrientes, principalmente vitamina $\mathrm{C}$, beta caroteno e folato, e o uso de anticoncepcionais ${ }^{3}$.

A principal estratégia utilizada para detecção precoce da doença no Brasil é através da realização do exame citopatológico preventivo do câncer do colo do útero, conhecido popularmente como exame de Papanicolaou. O exame pode ser realizado nos postos ou unidades de saúde que tenham profissionais capacitados para realizá- $\operatorname{los}^{4}$.

Embora tenha sido um dos primeiros países no mundo a realizar a citologia do Papanicolau, dados mostram que os índices de mortalidade por câncer cervical não registraram nenhuma queda nos últimos vinte anos, sendo que no ano de 2000, o câncer de colo uterino foi responsável pelo óbito de 3.953 mulheres em todo território nacional, fato que pode estar ligado à baixa cobertura de citologia oncótica. A estimativa é de que em 2006 tenham surgido 19.260 novos casos por câncer do colo do útero em todo o país ${ }^{1}$.

Dentre todos os tipos de câncer, este é o que apresenta um dos mais altos potenciais de prevenção e cura, chegando perto de 100\%, quando diagnosticado precocemente, podendo ser tratado em nível ambulatorial em cerca de $80 \%$ dos casos. O controle dessa doença dispõe de tecnologia de baixo custo para a prevenção e detecção através do esfregaço do citopatológico, porém para a obtenção de um impacto epidemiológico na freqüência do câncer uterino é necessário 85\% de abrangência da população feminina ${ }^{5}$. Porém, estima-se que cerca de $40 \%$ das mulheres nunca tenham sido submetidas ao exame citopatológico ${ }^{6}$.

O exame citopatológico consiste na análise das células oriundas da ectocérvice e da endocérvice que são extraídas por raspagem do colo do útero ${ }^{7}$.

A coleta do exame é realizada durante uma consulta ginecológica de rotina, após a introdução do espéculo vaginal, sem colocação de nenhum lubrificante. Normalmente não é doloroso, mas um desconforto variável pode acontecer, de acordo com a sensibilidade individual de cada paciente. As mulheres devem ser previamente orientadas a não terem relações sexuais ou fazerem uso de duchas, medicamentos ou exames intravaginais durante as 48 horas que precedem o exame a fim de garantir a eficácia dos resultados.

Inicialmente, um exame deve ser feito a cada ano e, caso dois exames seguidos, no intervalo de um ano, apresentem resultado normal, o exame pode passar a ser feito a cada três anos. No caso de mulheres histerectomizadas que comparecerem para a coleta, deve ser obtido um esfregaço de fundo do saco vaginal. Mulheres grávidas também podem realizar o exame. Neste caso, são coletadas amostras do fundo do saco vaginal posterior e da ectocérvice, mas não da endocérvice, para não estimular contrações uterinas.

Toda mulher que tem ou já teve atividade sexual deve submeter-se a exame preventivo periódico, especialmente se estiver na faixa etária dos 25 aos 59 anos de idade.

É fundamental que os serviços de saúde orientem sobre o que é, e qual a importância do exame preventivo, pois a sua realização periódica permite reduzir a mortalidade por câncer do colo do útero na população de risco $^{8}$. 
A detecção precoce do câncer uterino em mulheres assintomáticas, por meio do citopatológico, permite a detecção das lesões precursoras da doença em estágios iniciais, antes mesmo do aparecimento dos sintomas ${ }^{9}$.

A prevenção primária do câncer do colo do útero pode ser realizada através do uso de preservativos durante a relação sexual, uma vez que a prática de sexo seguro é uma das formas de evitar o contágio pelo Papiloma Vírus Humano $(\mathrm{HPV})^{3}$.

\section{O HPV}

Desde 1992, a Organização Mundial de Saúde considera que a persistência da infecção pelo $\mathrm{Pa}$ piloma Vírus Humano representa o principal fator de risco para o desenvolvimento da doença. Sabe-se também que a infecção pelo HPV é essencial, mas não suficiente para a evolução do câncer de colo uterino.

A prevalência do HPV na população em geral é alta (5 a 20\% das mulheres sexualmente ativas mostram positividade em testes moleculares) e este aumento tem sido sentido a partir de 1960, coincidente com o aumento do uso de contraceptivos orais, diminuição do uso de outros métodos de barreira e avanço tecnológico nos métodos diagnósticos.

O HPV estabelece relações amplamente inofensivas e a maioria das infecções passam despercebidas, regredindo de maneira espontânea. São várias as formas de interação com o organismo humano. $\mathrm{Na}$ forma latente, a mulher não apresenta lesões clínicas, e a única forma de diagnóstico é a molecular. Quando a infecção é subclínica, a mulher não apresenta lesões diagnosticáveis a olho nu, e o diagnóstico pode ser sugerido a partir da citopatologia, colposcopia, ou histologia. Na forma clínica, existe uma lesão visível macroscopicamente, representada pelo condiloma acuminado, com quase nenhuma potencialidade de progressão para o câncer.

Segundo Andreoli ${ }^{7}$, é comum no resultado da prevenção do câncer do colo uterino ou mesmo nas colposcopias a suspeita de HPV. Isso reflete comportamento sexual distinto. Estudos recentes mostram ainda que o papiloma vírus humano (HPV) tem papel importante no desenvolvimento da displasia das células cervicais e na sua transformação em células cancerosas. Este vírus está presente em mais de 90\% dos casos de câncer do colo do útero.

Observa-se hoje, que alguns pacientes infectados, são inativos sexuais ou mesmo crianças, indicando mais de uma via de contaminação além da sexual. No homem o diagnóstico é realizado também por coleta de exames para citologia, primeiro jato urinário e peniscopia. Os achados de ação viral em citologia propõem uma avaliação mais acurada pela colposcopia, investigando as áreas preferenciais do canal cervical, as zonas de substituição ou transformação.

Por ser considerada Doença Sexualmente Transmissível (DST), implica maior incidência entre os que praticam relações sexuais com múltiplos parceiros. Entre as mulheres fumantes observa-se uma maior incidência. Em pessoas imunodeprimidas, sejam por doenças adquiridas como AIDS ou pacientes em uso crônico de corticóides ou imunossupressores.

Verifica-se a relevância de uma mudança estratégia no combate ao câncer de colo uterino, combinando ações preventivas, de promoção e proteção à saúde, com medidas diagnósticas precoces, com objetivo principal de reduzir a mortalidade por este tipo de câncer.

É neste contexto que o presente estudo foi proposto, com o objetivo de promover palestras voltadas para a educação em saúde e desenvolver uma reflexão crítica entre mulheres de diferentes faixas etárias sobre câncer do colo uterino. Principalmente estimulando a identificação de sintomas e a procura pelo serviço de prevenção adequado, oferecido pela Secretaria Municipal de Saúde.

\section{Educação em saúde}

A promoção da saúde visa assegurar a igualdade de oportunidades e proporcionar os meios que permitam a todas às pessoas realizar completamente seu potencial de saúde. Os indivíduos e as comunidades devem ter oportunidade de conhecer e controlar os fatores determinantes da sua saúde. Ambientes favoráveis, acesso à informação, habilidades para viver melhor, bem como oportunidades para fazer escolhas mais saudáveis, estão entre os principais elementos capacitantes. Os profissionais e os grupos sociais, assim como as equipes de saúde, têm a responsabilidade de contribuir para a mediação entre os diferentes interesses, em relação à saúde, existentes na sociedade ${ }^{8}$.

Os programas ou atividades de promoção da saúde tendem a concentrar-se em componentes educativos, primariamente relacionados com riscos comportamentais passíveis de mudanças, que estariam, pelo menos em parte, sob o controle dos próprios indivíduos. Por exemplo, o 
hábito de fumar, a dieta, as atividades físicas, a direção perigosa no trânsito. Nessa abordagem, fugiriam do âmbito da promoção da saúde todos os fatores que estivessem fora do controle dos indivíduos ${ }^{8}$.

O desenvolvimento de habilidades e atitudes pessoais favoráveis à saúde em todas as etapas da vida encontra-se entre os campos de ação da promoção da saúde. Para tanto, é imprescindível a divulgação de informações sobre a educação para a saúde, o que deve ocorrer no lar, na escola, no trabalho e em muitos outros espaços coletivos.

Particularmente em países como o Brasil e outros da América Latina, a péssima distribuição de renda, o analfabetismo e o baixo grau de escolaridade, assim como as condições precárias de habitação e ambiente têm um papel muito importante nas condições de vida e saúde.

É necessário estimular a população feminina a determinar suas próprias metas de saúde e comportamentos, a aprender sobre saúde e doenças, com estratégias de intervenção e de apoio, com aconselhamentos e supervisão contínua. As áreas de interesse especial na promoção de saúde da mulher incluem a higiene pessoal, estratégias para detectar e prevenir doenças, em particular as DSTs, tais como infecção pelo vírus da imunodeficiência humana (HIV), e aspectos relacionados à sexualidade e ao funcionamento sexual, como contracepção, menopausa, entre outros ${ }^{9}$.

$\mathrm{O}$ que de fato faz-se importante em apostar em prevenção, primeiramente é o melhoramento da qualidade de vida da população e a diminuição com gastos médico-hospitalares. Investir em prevenção e conscientização da população é menos oneroso que o tratamento curativo dos diversos tipos de patologias, principalmente na rede pública de saúde, reduzindo assim os custos com internações, cirurgias e tratamentos. Para tanto é necessário que durante os exames e consultas essas mulheres sejam atendidas com respeito e dignidade, para que não se sintam envergonhadas ou constrangidas e acabem desistindo de realizar futuros exames e consultas.

O desenvolvimento de habilidades e atitudes pessoais favoráveis à saúde em todas as etapas da vida encontra-se entre os campos de ação da promoção da saúde. Para tanto, é imprescindível a divulgação de informações sobre a educação para a saúde, o que deve ocorrer no lar, na escola, no trabalho e em muitos outros espaços coletivos.
Particularmente em países como o Brasil e outros da América Latina, a péssima distribuição de renda, o analfabetismo e o baixo grau de escolaridade, assim como as condições precárias de habitação e ambiente têm um papel muito importante nas condições de vida e saúde.

\section{Caminho metodológico}

O presente estudo foi realizado em cinco diferentes localidades do município de Santo Ângelo/RS envolvendo mulheres com idade superior a 15 anos, que assistiram às palestras durante encontros que se realizaram nos grupos de convivência dos quais estas participavam, no período de fevereiro a junho de 2007.

Nos encontros procurou-se desenvolver uma reflexão crítica entre as mulheres de diferentes faixas etárias sobre câncer do colo uterino. Além disso, a procura pelo serviço de prevenção e assistência adequadas também eram estimulados, tendo em vista a existência desse tipo de atendimento oferecido gratuitamente pela Secretaria Municipal de Saúde do município. Foi realizado também um estudo de levantamento do perfil da saúde sexual das mulheres participantes através da aplicação de um questionário padrão. Os questionários eram constituídos por perguntas abertas e fechadas e de alternativas fixas e diretas, para evitar desvios do entrevistado, que segundo Henri $^{10}$, é uma técnica de pesquisa eficiente na elaboração do perfil de um grupo específico.

O presente trabalho de pesquisa foi aprovado pelo Comitê de Ética em Pesquisa da Universidade Regional Integrada do Alto Uruguai e das Missões.

O critério de seleção das entrevistadas, quanto ao perfil de saúde sexual, foi constituído a partir do desejo individual de participar do estudo.

Ao final dos encontros/palestras as participantes que aceitaram participar do estudo receberam os questionários que foram os instrumentos de investigação da pesquisa, os quais foram respondidos com autorização prévia, mediante assinatura do Termo de Consentimento Livre e Esclarecido, elaborado segundo resolução nº 196, de 10/10/1996, do Conselho Nacional de Saúde ${ }^{11}$. O sigilo e o anonimato das identidades foram assegurados às participantes, solicitando permissão para o uso dos dados coletados.

Os resultados obtidos foram analisados por estatística descritiva no programa Excel. 


\section{Resultados e discussão}

Foram realizados cinco encontros/palestras, abrangendo um total de sessenta mulheres com idade entre 15 e 60 anos, sendo a idade média de 37,4 anos (Tabela 1).

O grupo estudado apresentou faixa etária mais prevalente de 20 a 30 anos (47\%) e era constituída principalmente por mulheres casadas $(45 \%)$ e brancas $(82 \%)$. Quanto ao nível de escolaridade observou-se que a maioria das mulheres (49\%) possuía nível superior (Tabela 2).

\section{Periodicidade do Exame Preventivo}

Quanto aos aspectos de saúde, a maioria das mulheres (99\%) relatou ciclos menstruais de caráter regular. Conforme observado, 2 mulheres (3\%), nunca haviam realizado o exame citopatológico e 7 (12\%), não tinham conhecimento do mesmo. Quanto à periodicidade com que as mulheres realizavam o exame de prevenção, das 60 entrevistadas, 41 (68\%) relataram estar em dia com o citopatológico, 19 (32\%) não estavam e, deste contingente, há aquelas que só procuraram ou procuram atendimento a partir de sintomas.

Entre as mulheres que se recordavam de quando haviam realizado o exame, a maior parte delas ( $\mathrm{n}=41 ; 68 \%)$ havia realizado no intervalo de 1 ano. Ainda com relação à faixa etária, pôde-se observar que as mulheres acima de 30 anos foram as que mais freqüentemente realizavam $o$ exame citopatológico.

Independentemente da realização ou não do citopatológico, todas as mulheres foram inquiridas sobre o conhecimento da necessidade da rea-

\begin{tabular}{|c|c|}
\hline 15 à 20 anos & $9(15 \%)$ \\
\hline 20 à 30 anos & $28(47 \%)$ \\
\hline 30 à 40 anos & $13(22 \%)$ \\
\hline 40 à 50 anos & $8(13 \%)$ \\
\hline 50 à 60 anos & $2(3 \%)$ \\
\hline
\end{tabular}

Tabela 2. Escolaridade

\begin{tabular}{lr}
\hline Ensino fundamental Incompleto & $9(15 \%)$ \\
Ensino fundamental Completo & $2(3 \%)$ \\
Ensino Médio Incompleto & $2(3 \%)$ \\
Ensino Médio Completo & $18(30 \%)$ \\
Nível superior & $29(49 \%)$ \\
\hline
\end{tabular}

lização do mesmo e de sua periodicidade, sendo reconhecidos como intervalos aceitáveis de 1 a 2 anos, e com a finalidade de prevenção do câncer.

Quanto ao número de parceiros, a maioria das mulheres $(n=42 ; 70 \%)$ relataram ter relações sexuais com no máximo cinco parceiros durante toda a sua vida sexual (Figura 1).

Quanto à idade da primeira relação sexual das entrevistadas, 49 mulheres (82\%) responderam ter tido a sua primeira relação entre os $12 \mathrm{e}$ 20 anos, e $(11 ; 18 \%)$ tiveram a primeira relação entre os 20 e 30 anos.

É importante salientar que em alguns grupos etários jovens, principalmente naqueles compreendidos entre 20 e 30 anos, a incidência tem aumentado não só pelo acesso a serviços de saúde, determinado por algum grau de desenvolvimento socioeconômico, mas também pela maior exposição a agentes sexualmente transmissíveis e ao uso inadequado de métodos contraceptivos de barreira ${ }^{12}$.

\section{Filhos e o papel social da mulher}

Das entrevistadas, $43(72 \%)$ trabalhavam fora, possuindo renda própria, e 17 (28\%) não. Isso mostra que uma importante alteração tem ocorrido no campo de trabalho das mulheres brasileiras e que à medida que elas aumentam sua presença, ocorrem alterações em seus papéis, estilo de vida e padrões familiares.

A maioria das entrevistadas não tinha filhos, totalizando 30 mulheres. Dentre aquelas que tinham, aparecem em primeiro lugar 16 mulheres com a idade do primeiro parto dos 20 aos 30 anos, em segundo lugar, 10 mulheres com idade dos 14 aos 20 anos e 3 mulheres que tiveram filhos entre os 30 e 40 anos de idade (Tabela 3).

Mudanças ao longo do tempo incluíram o retardamento da gestação e da criação de filhos até que uma carreira esteja estabelecida. Vários

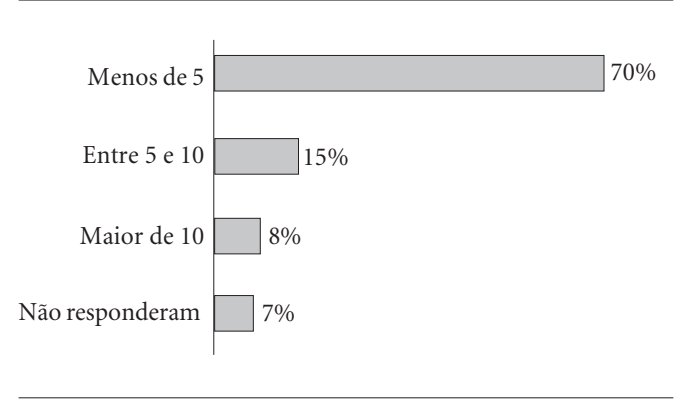

Figura 1. Número de parceiros sexuais. 
métodos de contracepção tornam possível esta opção. Porém ainda existe um grande percentual de mulheres sem informação, com cultura restrita e vivendo em condições de miséria, onde o planejamento familiar inexiste ${ }^{13}$.

Quanto aos métodos contraceptivos, os mais relatados foram a pílula anticoncepcional $(\mathrm{n}=36$; $48 \%)$, e o preservativo masculino $(n=20 ; 27 \%)$ (Figura 2).

A análise da associação, se é que existe, entre o uso de contraceptivos orais e o risco de câncer do colo do útero é feita com dificuldades. Os contraceptivos orais são usados por mulheres sexualmente ativas e que, em menor probabilidade, usam métodos de barreira, sendo por isto mais expostas ao risco de contrair HPV. Em compensação, essas mulheres comparecem mais ao ginecologista, tendo maior possibilidade de serem rastreadas para o câncer do colo do útero.

Em relação ao histórico prévio de DSTs, 51 mulheres $(87 \%)$ responderam nunca ter adquirido qualquer tipo de DST, $5(8 \%)$ responderam já terem contraído DST, e 4 (5\%) não responderam. A presença de uma DST, além de aumentar o risco de contrair e transmitir o HIV, também pode provocar o aparecimento de feridas e de inflamações nas mucosas e pele dos genitais, que

Tabela 3. Idade do primeiro parto

$\begin{array}{lr}\text { Não tinham filhos } & 30(49 \%) \\ 14 \text { aos } 20 \text { anos } & 10(17 \%) \\ 20 \text { aos } 30 \text { anos } & 16(27 \%) \\ 30 \text { aos } 40 \text { anos } & 4(7 \%)\end{array}$

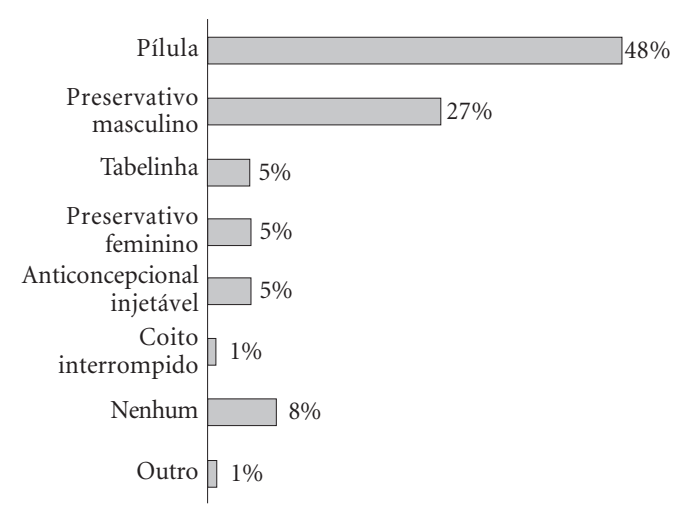

Figura 2. Métodos contraceptivos usados. se não forem tratadas adequadamente podem tornar-se lesões pré - cancerosas, levando futuramente ao câncer de colo uterino.

Quanto à presença de corrimentos, $43 \mathrm{mu}$ lheres $(72 \%)$ responderam não apresentar, enquanto 17 (28\%) disseram apresentar algum tipo, e quanto ao aparecimento de sangramento vaginal houve relato de apenas um caso.

Um dos problemas mais comuns que afeta a saúde da mulher é o corrimento vaginal também chamado de vaginite. É uma das causas mais freqüentes de visita ao médico ginecologista. Caracteriza-se por uma irritação vaginal ou um corrimento anormal que pode ou não ter cheiro desagradável. Pode haver também coceira ou ardor na vagina, ou vontade mais freqüente de urinar. Os corrimentos podem ser causados por infecções vaginais, infecções cervicais ou do colo do útero, ou ainda por doenças sexualmente transmissíveis $1^{14}$.

Quanto à presença de dor nas relações sexuais, 24 mulheres (40\%) disseram nunca ter sentido dor, e 36 (60\%) relataram já ter apresentado algum tipo de dor ou desconforto.

É importante atentar ao fato de que os principais sintomas aparecem quando o câncer invade outros tecidos ou órgãos. Alguns sinais e sintomas possíveis de displasia ou câncer cervical podem estar relacionados a pequenos sangramentos fora do período menstrual, menstruações mais longas e volumosas que o usual, sangramento após relação sexual, ducha ou exame vaginal, dor durante a relação, sangramento após a menopausa, e aumento de secreção vaginal. Estes são sinais simples aos quais a mulher deve estar atenta e procurar serviço médico especializado, pois quando apresentam alguns destes sintomas muitas mulheres tendem a ignorá-los por parecer que estão relacionados com condições pouco sérias, ou por tratarem-se de algo normal do corpo da mulher. Quanto mais tempo se leva para diagnosticar o câncer de colo e mais tempo se demora a iniciar o tratamento, menores são as chances de cura.

Em relação à história familiar de câncer do colo uterino na família, houve a presença de 13 relatos positivos $(22 \%)$, sendo que as demais negaram ou desconheciam sua história familiar. Mães e tias correspondem ao grau de parentesco mais afetado, seguido de irmãs, com duas citações e primas e avós com uma citação cada.

Os principais motivos pelos quais as mulheres entrevistadas procuram realizar o exame citopatológico são: a questão da dor abdominal, sangramento após as relações sexuais, menstru- 
ações irregulares, solicitação de algum médico, presença de leucorréia e medo, por terem familiares, amigas, vizinhas, que tiveram a doença.

\section{Prevenção do câncer de colo de útero na percepção da mulher}

Verificou-se que durante os encontros as mulheres percebem o exame de prevenção como uma forma de se cuidar. Algumas demonstravam preocupação e interesse em saber suas condições de saúde. Apesar de reconhecer, no entanto, a importância da prevenção e preservação da saúde como possibilidade de uma vida saudável, algumas mulheres disseram que às vezes em que buscaram assistência foi por apresentarem o aparecimento de sintomas.

\section{Expressão de sentimentos: a vergonha e o medo}

Ainda, verifica-se por parte de algumas mulheres, resistência à procura pelo exame ginecológico simplesmente pelo constrangimento ou por desconhecerem a importância do mesmo na prevenção do câncer. Além disso, existem ainda, aquelas que consideram sintomas importantes do câncer de colo de útero, como uma coisa normal de toda mulher. Aqui, além das dificuldades intrínsecas de cada mulher, existem outras que também interferem no acesso aos serviços de saúde: o exame como empecilho para as obrigações sexuais da mulher para com o marido, o baixo poder aquisitivo, o que provoca uma série de dificuldades para se ausentar de casa.

Dentre os motivos pelos quais não realizavam o exame citopatológico aparece também o medo. Pode-se dizer que o medo é um sentimento de inquietação diante de um perigo real ou imaginário. Myra e Lopez ${ }^{15}$ compreendem que o medo age como sinal condicionante e antecipador de medos de sofrimento, caso tome proporções altas. O medo é desencadeado a partir de uma situação concreta, presente e maléfica. Algumas participantes relataram que o medo está relacionado com o exame, ou seja, com a realização deste, e à expectativa de terem alguma coisa, mas, a falta de informação em muitos casos funciona como indutor de nervosismo, ansiedade e medo, conforme depoimentos.

\section{Considerações finais}

O estudo revelou que, mesmo enfrentando dificuldades e medo, a grande maioria das mulheres procura o serviço de saúde para se submeter ao exame de prevenção do câncer cérvico-uterino. A motivação para realizar esse exame está vinculada ao aparecimento de sintomas, ao hábito de cuidar-se e/ou na preocupação com sua condição de saúde.

Muitas mulheres deixaram claro que, em relação ao exame, sentem-se algumas vezes constrangidas, envergonhadas, com medo da dor ou da ocorrência de sangramento durante o mesmo, e principalmente quanto à positividade do resultado.

Foram citados também alguns outros impedimentos como baixo poder aquisitivo, o fato de cuidar de filhos e marido, e desinformação sobre o exame.

É fato que a mortalidade por câncer do colo do útero é evitável, uma vez que as ações para seu controle contam com tecnologias para o diagnóstico e tratamento de lesões precursoras, permitindo a cura em $100 \%$ dos casos diagnosticados na fase inicial.

É, portanto, fundamental que haja mecanismos por meio dos quais mulheres motivadas a cuidar de sua saúde encontrem uma rede de serviços quantitativamente e qualitativamente capaz de suprir essa necessidade em todo o país.

De uma maneira geral, o sucesso desses mecanismos está relacionado a fatores como cobertura efetiva da população de risco, qualidade na coleta e interpretação do material, tratamento e acompanhamento adequados.

Por esta razão, consideramos relevante a educação permanente em saúde, atividades educativas junto às mulheres, parcerias entre serviços de saúde e universidades e/ou escolas e organizações que trabalhem com esse tema e que possam promover a atenção para prevenção do câncer do colo do útero. Deve-se priorizar atividades de educação para o diagnóstico precoce e rastreamento em mulheres sintomáticas e assintomáticas, respectivamente, além da garantia de acesso aos métodos de diagnóstico e tratamento adequados.

Os postos de saúde na sua grande maioria oferecem o exame preventivo do câncer de colo uterino. A garantia do oferecimento do exame citopatológico ao maior número possível de mulheres é muito importante, pois, muitas vezes estas deixam de realizar por falta de locais para atendimento, e isso contribuirá para uma redução significativa de óbitos de mulheres muitas 
vezes chefes de família, que acabam deixando filhos e companheiros, perda que pode provocar crises sociais e emocionais sérias, uma vez que o sofrimento se instala, provocando um desequilíbrio familiar.

$\mathrm{O}$ acesso ao atendimento básico, quando facilitado e ágil, pode estimular as mulheres a procurarem os serviços de saúde.

\section{Colaboradores}

MR Casarin trabalhou na coleta e análise dos dados e preparo inicial dos manuscritos, JCE Piccoli, na redação e discussão teórica, na interpretação dos dados e trabalhou na aprovação da versão a ser publicada.

\section{Referências}

1. Brasil. Ministério da Saúde (MS). Instituto Nacional do Câncer (INCA). Dados sobre câncer de colo do útero. [texto na Internet]. 2006. [acessado 2006 set 28]. Disponível em: www.inca.org.br

2. Duavy LM, Batista FLR, Jorge MSB, Santos JBF. A percepção da mulher sobre o exame preventivo do câncer cérvico-uterino: estudo de caso. Cien Saude Colet 2007; 12(3):733-742.

3. Oliveira MMHN, Silva AAM, Brito LMO, Coimbra LC. Cobertura e fatores associados à não realização do exame preventivo de Papanicolaou em São Luís, Maranhão. Rev Bras Epidemiologia 2006; 9(3):325-334.

4. Diógenes MAR, Rezende MDS, Passos NMG. Prevenção do Câncer: Atuação do enfermeiro na Consulta de enfermagem. $2^{\text {a }}$ ed. Fortaleza: Pouchain Ramos Gráfica; 2001.

5. Brasil. Ministério da Saúde. Instituto Nacional do Câncer. Incidência de câncer no Brasil. Estimativa/ 2006. Brasília: Instituto Nacional do Câncer. [acessado 2006 out 13]. Disponível em: http://www.inca. gov.br/estimativas

6. Tenconi P, Becker T, Pasini, A, Haas P. Estudo da Incidência de Câncer de Colo de Útero nas Regiões da Grande Florianópolis e Sul do Estado de Santa Catarina e Análise da Metodologia Utilizada Para Realização do Exame. Rev. NewsLab 2000; 40:164178.

7. Andreoli TE, Carpenter CCJ, Griggs RC, Loscalzo J. Medicina Interna Básica. Rio de Janeiro: Guanabara Koogan S/A; 1998.
8. Czeresnia, D, Freitas CM, organizadores. Promoção da saúde: conceitos, reflexões, tendências. Rio de Janeiro: Fiocruz; 2003.

9. Ações em saúde: Saúde da Mulher. Normas técnicas e Operacionais. Porto Alegre: SSMA/RS; 1997.

10. Henri J. et al. Pesquisa em Enfermagem. Novas psicologias Aplicadas. Rio de Janeiro: Guanabara Koogan; 1998.

11. Brasil. Ministério da Saúde (MS). Conselho Nacional de Saúde. Resolução no 196, de 10 de outubro de 1996. Brasília: CONAS; 1996.

12. Oliveira MM, Silva ENF, Pinto IC, Coimbra VCC. Câncer cérvico uterino: um olhar crítico sobre a prevenção. Rev. Gaúcha Enfermagem 2004; 25(2):176183.

13. Frederickson HL, Hang LW. Segredos em Ginecologia e Obstetrícia. 2a ed. Porto Alegre: Artmed; 2000.

14. Smeltzer SC, Bare BG. Brunner \& Suddarth: Tratado de Enfermagem Médico Cirúrgico. v. 8. 8a ed. Rio de Janeiro: Guanabara Koogan S/A; 2000.

15. Myra y Lopez E. Os quatro gigantes da alma: o medo, a ira, o dever, o amor. Rio de Janeiro: José Olímpio; 1996.

Artigo apresentado em 12/07/2008

Aprovado em 05/12/2008

Versão final apresentada em 10/01/2009 